\title{
LA GENTRIFICACIÓN EN COSTA RICA: ELEMENTOS PARA SU ESTUDIO Y COMPRENSIÓN
}

\section{THE GENTRIFICATION IN COSTA RICA: CONTRIBUTIONS FOR STUDY AND UNDERSTANDING ${ }^{1}$}

\author{
Wendy Molina Varela*
}

RESUMEN

Ante la ausencia de investigaciones previas sobre la gentrificación en Costa Rica, el objetivo de este artículo es plantear algunos elementos de análisis para el abordaje del tema en el país. Para ello se analiza el debate que existe alrededor del concepto, su aplicación en otros países latinoamericanos y sus hallazgos, así como, las características del desarrollo urbano en Costa Rica desde inicios del siglo xx. El resultado es un encuadre teórico y de antecedentes del tema, así como, una necesaria discusión acerca del abordaje y las preguntas para una posible investigación posterior. La principal conclusión es que en Costa Rica existe un modelo de gobernanza urbana neoliberal, en el que el Estado permite la acción del sector privado en el crecimiento de la ciudad, pero no la dirige o financia directamente en los términos de un modelo de gobernanza urbana empresarial, y por lo tanto, hasta ahora no ha habido condiciones favorables para la renovación urbana y procesos asociados como la gentrificación en zonas céntricas. Sin embargo, no se descarta que la gentrificación se esté produciendo en otras condiciones y con otras características.

PALABRAS CLAVE: COSTA RICA * DESARROLLO URBANO * ZONA METROPOLITANA * DESIGUALDAD SOCIAL * MIGRACIÓN INTERNA

\section{ABSTRACT}

In the absence of previous research on gentrification in Costa Rica, the objective of this article is to propose some elements of analysis to address the issue in this country. For this, the debate that exists around the concept, its application in other Latin American countries and its findings, as well as the characteristics of urban development in our country since the beginning of the 20th century is analyzed. The result is a theoretical framework and background of the subject, as well as a necessary discussion about the approach and the questions for a possible subsequent investigation. The main conclusion

1 Esta investigación forma parte del "Concurso Estancias de Investigación 2017-2018", del Instituto de Investigaciones Sociales (IIS), Universidad de Costa Rica (UCR). Código 725-B7-791. 
is that in Costa Rica there is a model of neoliberal urban governance, in which the State allows private sector action in the growth of the city, but does not directly direct or finance it in terms of an urban governance model. business, and therefore, until now there have been no favorable conditions for urban renewal and associated processes such as gentrification in downtown areas. However, it is not ruled out that gentrification is taking place in other conditions and with other characteristics.

KEYWORDS: COSTA RICA * URBAN DEVELOPMENT * METROPOLITAN AREAS * SOCIAL INEQUALITY * INTERNAL MIGRATION

\section{INTRODUCCIÓN}

El concepto gentrificación, acuñado originalmente por Ruth Glass (1964), para describir un proceso de rehabilitación acelerada de casas victorianas, el aumento en el precio de las propiedades y el recambio poblacional de la clase trabajadora por una clase media en proceso migratorio desde la periferia hacia Londres, se define como la reestructuración espacial de un área urbana que trae consigo el desplazamiento de residentes de bajos ingresos por la llegada de estratos socioeconómicos superiores (Glass, 1964 citada en López, 2016).

A partir de esta fecha, la relación entre vivienda y lucha de clases en Londres (particularmente en Islington, donde vivía Glass) se convirtió en su tema de investigación a lo largo de su vida; $y$ con esta línea investigativa diversos autores desarrollaron todo un campo de estudios comparados bajo el término "gentrificación", tanto en el mundo anglosajón como en el resto de las ciudades del mundo $y$ Latinoamérica.

La aplicación posterior que se ha hecho de este concepto, ha generado un fuerte debate, pues por un lado se cuestionan las generalizaciones que provienen de trabajos y teorías urbanas propias de la realidad anglosajona, $y$ por otro lado, se discute la aplicación indiscriminada a una gran diversidad de procesos de urbanización (de naturaleza comercial, residencial, turística; $y$ de tipo renovación, redesarrollo, verticalización, etc.), en donde no siempre se presentan todas las características del fenómeno que originalmente describió Glass (Rojo, 2016).
Por otra parte, más allá del debate conceptual, se encuentra en la literatura una gran diversidad analítica en la identificación o explicación de la gentrificación de acuerdo con el énfasis o foco explicativo en el que se sitúa el trabajo, se encuentran estudios sobre: 1) el sujeto gentrificador; 2) el agente gentrificador, sea el Estado o el Mercado; 3) las zonas de la ciudad (centro, periferia, ruralidad en transición), o 4) las consecuencias de la gentrificación y las formas de desplazamiento de poblaciones originales (Rojo, 2016). Siguiendo estos énfasis temáticos, se tiene una gran diversidad de metodologías de identificación o medición del fenómeno, tan diferentes como los casos a los que se aplican (Duque, 2010; Maloutas, 2011; Rojo, 2016; Rubiales, 2014).

En el primer apartado del presente artículo, se resume ese debate partiendo de la siguiente premisa: que a nivel teórico existen básicamente dos corrientes teóricas sobre cómo explicar la naturaleza del fenómeno o sus causas, por una parte, la corriente macro (basada en la comprensión de la oferta inmobiliaria), y por otra, la corriente micro (basada en la comprensión de la demanda inmobiliaria) (Lees, Slater y Wyly, 2008).

En un segundo apartado, se desarrolla el debate entorno al abordaje del estudio de la gentrificación en Latinoamérica. Debate que sigue la senda de una discusión entre el enfoque estructuralista o postcolonial, desde los cuales se explica la pertinencia de aplicar el concepto gentrificación en Latinoamérica, así como, sus implicaciones $y$ alcances. 
En un tercer apartado, se plantean algunas consideraciones para el estudio de la gentrificación en Costa Rica: los factores históricos, geográficos, socioeconómicos y políticos de la historia de la Gran Área Metropolitana (GAM); y con ello, se delinean los elementos fundamentales para la comprensión de la gentrificación en el país desde un punto de vista histórico y particular.

Finalmente, en un cuarto apartado de discusión, se desarrolla el objetivo central de este trabajo, a saber, retomar el debate teórico expuesto anteriormente, exponer las particularidades de la GAM $y$ construir un encuadre para el caso de Costa Rica. Para ello se establece el marco teórico adecuado y algunas consideraciones del contexto particular, y desde ahí, se problematiza acerca del abordaje del estudio de la gentrificación en nuestro país y se plantean interrogantes para una investigación posterior.

\section{EL DEBATE SOBRE EL CONCEPTO GENTRIFICACIÓN}

En el presente apartado se realiza un recorrido de los principales autores en el tema de la gentrificación, pero poniendo énfasis en las contribuciones más sintéticas y en los mayores aportes, esto con el fin de cumplir con un estudio exhaustivo, pero sobre todo con el objetivo de delimitar y definir un marco teórico adecuado para el estudio de la gentrificación en Costa Rica.

Una de las contribuciones más sintéticas de la teoría y el debate existente alrededor de este concepto y sus aplicaciones, lo realiza Lees et ál. (2008) en su trabajo Gentrification, en el cual se revisa de forma exhaustiva la literatura existente sobre el tema desde su primera aparición en los años 1960. Para estos autores, en diversos trabajos del mismo corte, el debate alrededor del concepto de gentrificación básicamente gira alrededor de dos corrientes teóricas: el enfoque de la demanda (microeconómico) y el enfoque de la oferta (macroeconómico) (Lees, Slater y Wyly, 2008; Lees, Shin y López-Morales, 2015 y 2016).

El enfoque microeconómico analiza la gentrificación a partir de la demanda inmobiliaria. Explica que en países de economías postindustriales se producen cambios en la estructura ocupacional que generan una "nueva clase media", lo que trae consigo cambios culturales importantes en relación con los gustos $y$ preferencias de consumo, entre estas, el consumo de vivienda asociado a estilos de vida urbanos (Betancur, 2014; Lees et ál, 2008; López, 2009; Maloutas, 2011).

A su vez, se oponen a lo que consideran la difusión del concepto gentrificación desde el norte al sur, $y$ por lo tanto, a su aplicación fuera del contexto anglosajón. Esto último implica rechazar desde el enfoque basado en la demanda la idea de que en el sur pueda verificarse la existencia de esa "nueva clase media" que constituye el sujeto gentrificador (la demanda), el que fuera identificado por David Ley (1996) como una clase media, que habitualmente se encuentra soltera $y$ sin hijos, alrededor de los 35 años de edad o menos, $y$ que su condición de gentrificador viene dada por sus niveles de ingreso, asociados a ser empleados en el sector de servicios avanzados.

La segunda corriente (macroeconómica), explica la gentrificación a partir de la oferta inmobiliaria. Lees et ál. (2008) explican, retomando principalmente los aportes de Neil Smith (1979, 1996, 2012 y 2015) y David Harvey (1973, 1985 y 2013), que la brecha en la renta, es decir, el diferencial entre la renta futura potencial y la renta actual capitalizada, produce un valle en la curva de valor del suelo que resulta de gran beneficio potencial para los desarrolladores inmobiliarios (rent gap o captura de brecha de renta de suelo).

Para estos autores, la gentrificación se basa en la captura y acumulación de esta brecha de renta por parte de las empresas inmobiliarias (verdadero sujeto gentrificador desde su perspectiva), proceso que a su vez implica la desposesión y expulsión de los habitantes históricos de un área determinada.

En palabras de Harvey, la renta de monopolio surge porque ciertos agentes sociales pueden obtener una mayor corriente de ingresos durante un tiempo dilatado en virtud del control exclusivo sobre algún artículo. 
(...) Una situación en la que esta categoría cobra mayor importancia, es cuando algunos agentes controlan algún recurso, mercancía ó lugar con cualidades especiales, lo que les permite, en relación con cierto tipo de actividad, extraer rentas de monopolio de quienes desean usarlo. (...) En esas circunstancias, el precio de monopolio crea renta. (...) Se puede generar escasez apartando esa tierra, recurso o activo de su uso corriente $y$ especulando sobre su valor futuro (Harvey, 2013, p. 139-141).

Justamente, este proceso descrito anteriormente es impulsado, en el contexto de un proceso de gentrificación, por las mismas empresas inmobiliarias, entre otros mecanismos, mediante técnicas de marketing urbano que orientan la devaluación de la renta actual capitalizada y la sobrevaloración de la renta potencial (López et ál., 2014).

En un llamado a superar la dicotomía micro-macro, $y$ desde su perspectiva de aportar una síntesis, estos autores señalan que la revisión de la literatura producida sobre gentrificación deja claro que con el tiempo, más autores trabajan desde una postura síntesis que considera la estrecha relación entre la oferta y la demanda en la producción de la gentrificación. Asimismo, la forma en la que se relacionan entre sí lo micro y lo macro ha ido evolucionando en el tiempo, $y$ esto ha determinado un cambio en la escala de la gentrificación.

De esta forma, Lees et ál. (2008) determinan que se pueden identificar tres fases: una primera en la que la gentrificación aparece en ciudades postindustriales hasta finales de la década de los 60. En esta fase, la gentrificación obedece a políticas públicas orientadas a revertir el deterioro urbano, estas medidas se aplicaban a escala local y el fenómeno se presentaba a nivel barrial.

En una segunda fase, que ocurre durante los años 80 y 90 , ya no solo en ciudades centrales, la gentrificación es promovida por estrategias económicas mayores, participa capital financiero transnacional y se lleva a cabo por alianzas público-privadas con gran apoyo estatal mediante subsidios a la oferta.

En una tercera fase, la gentrificación se presenta a partir de la década de los 90 , tanto en ciudades del centro como en periferias mundiales, se caracteriza por su internacionalización con la participación de capital financiero mundial de mayor envergadura y operando con mayor escala geográfica. En esta fase, los instrumentos de crédito son más sofisticados y cubren tanto a la oferta como a la demanda, $y$ las políticas públicas de escala nacional y local dan gran apoyo mediante instrumentos de desarrollo urbano favorables a la gentrificación.

De esta forma, se habla de una "gentrificación planetaria" para describir un estadio en el que esta no solo se ha consolidado ya como un fenómeno global, sino que $-y$ gracias a esto-, ha mutado en múltiples formas, distanciándose del fenómeno original que observó Ruth Glass (1964). Actualmente, la gentrificación se presenta en contextos rurales, en otrora asentamientos informales, zonas turísticas y en espacios tanto urbanos como periurbanos; así como, en diferentes procesos de desarrollo urbano como la renovación urbana o el rescate patrimonial, en el redesarrollo o la verticalización, $y$ en lo que se ha dado en llamar supergentrificación, que consiste en la ocupación de los "superricos" de zonas previamente gentrificadas (López, 2009; Lees et ál., 2008; Lees et ál., 2015 y 2016).

El presente trabajo se adscribe a la propuesta teórica basada en el análisis de la oferta inmobiliaria, la que fundamentada en el concepto de brecha de renta identifica la captura de renta de suelo en una gran diversidad de casos, contextos y geografías a partir de una óptica de estudios comparados. Así, se entiende la gentrificación desde la explicación de las estructuras capitalistas que la generan (brecha de renta), pero considerando las particularidades sociológicas, culturales e institucionales que el fenómeno adquiere en cada lugar y siempre con el efecto de producir desplazamiento o exclusión de los segmentos sociales más bajos (Lees et ál., 2016).

Tal enfoque, admite $y$ se propone estudiar el fenómeno en sus múltiples manifestaciones. 
A este respecto, Janoschka sostiene que la aplicación del concepto a una gran diversidad de casos no significa una pérdida de rigor en la aplicación del término, sino que al contrario, lo fortalece como "un concepto válido para agrupar casos geográficamente variados que muestran la reestructuración espacial producto de formas también múltiples de neoliberalismo, y que por ello sirve para mostrar una diversidad de prácticas de apropiación del espacio" (Castrain y Janoschka, 2013, p.21).

Siguiendo esta línea de pensamiento, a continuación se resumen los principales hallazgos y aportes producto de estudios comparados entre ciudades, los que muestran la gran diversidad que existe en las manifestaciones del fenómeno de la gentrificación. En los estudios sobre la gentrificación en diferentes geografías se han identificado tanto procesos que se consideran más comunes, como la renovación urbana con expulsión de población, en el centro y pericentro (Contreras, 2011; Swanson, 2007); como procesos de renovación urbana con la particularidad de no presentar expulsión de la población y además ubicarse en la periferia (Sabatini, Robles y Vásquez, 2009); y así también la renovación urbana con expulsión de población pero fuera del centro (López, 2013); entre otras muy diversas tipologías del fenómeno y en diferentes zonas de la ciudad (Rojo, 2016).

Asimismo, Díaz (2013) ha identificado formas de desarrollo urbano que podrían formar parte del proceso de gentrificación, ya sea de forma complementaria o como fases intermedias de esta, siendo que no siempre ocurre de forma acelerada o lineal; estos son: invasiónsucesión, filtrado residencial, renovación urbana y redesarrollo. Todos estos fenómenos hacen referencia a transformaciones del espacio urbano a causa de la sustitución y expulsión de un grupo socioeconómico y sociocultural por parte de otro grupo.

Los procesos de invasión-sucesión se han estudiado en el pasado, principalmente en relación a la sustitución de unos grupos étnicos por otros en espacios urbanos concretos. Para un determinado enclave étnico-cultural, la pervivencia de este dependería de la incesante llegada de nuevos inmigrantes y la continuidad del escalón cultural entre este grupo y la sociedad de acogida. Esta es la primera fase de invasión liderada por los inmigrantes mas exitosos, que desean mejorar su estatus social desplazándose a un área de mayor prestigio (Díaz, 2013).

Por su parte, el filtrado residencial acontece dentro de la gentrificación, tanto como en otros procesos de transformación de hábitats sociales. Se trata de un concepto introducido por Hoyt, quien observaba cómo, en un contexto de laissez faire, los nuevos barrios eran casi siempre construidos para propietarios de altos ingresos, pero cuando las viviendas y los vecindarios envejecían, se producía el "filtrado hacia abajo" (down filtering) que hacía las residencias progresivamente más asequibles para grupos con menos recursos. El filtrado de abajo hacia arriba se produce claramente en el caso de la gentrificación, mientras el proceso inverso es vinculado por algunos autores a una fase previa, en la cual el barrio que sería gentrificado en el futuro va asociándose a un perfil cada vez más bajo, a medida que el sector entra en declive (Díaz, 2013).

Otro concepto cuya relación con la gentrificación puede resultar confusa es el de renovación urbana, la cual consiste en la transformación y mejora de la calidad urbana general por medio de la reparación o habilitación de edificios deteriorados o abandonados, así como de infraestructuras, espacios públicos y mobiliario urbano en general.

La mejora del entorno físico en algunos casos resulta ser un requisito fundamental para la gentrificación de un área, pues se trata de un proceso que opera en el mercado de viviendas $y$ que tiene como consecuencia fundamental la mejora de la edificación y la revalorización de un entorno dado. Esto implica que la renovación urbana puede ser causa de la gentrificación, al mismo tiempo que una de sus más evidentes consecuencias, pero no ha de confundirse con la misma (Díaz, 2013).

Finalmente, redesarrollo se denomina al fenómeno que no implica reparación o rehabilitación de estructuras deterioradas o antiguas, sino la construcción de nuevos edificios sobre suelos que ya estaban desarrollados (Díaz, 2013). "Algunos autores señalan la falta 
de desplazamiento en estos casos o la ausencia de los patrones estéticos y de consumo que han tendido a asociarse típicamente a la gentrificación. De esta forma, renovación urbana, redesarrollo y gentrificación son conceptos diferentes pero no excluyentes" (Díaz, 2013, p.12).

\section{LA GENTRIFICACIÓN EN LATINOAMÉRICA}

La discusión teórica acerca del estudio de la gentrificación en Latinoamérica gira básicamente en torno a una perspectiva estructuralista $y$ una perspectiva postcolonial, $y$ es a partir de estas dos posturas que se discute la aplicación del concepto en esta región y sus diferentes geografías de acuerdo con sus condiciones propias. Cabe destacar algunos elementos planteados por la perspectiva postcolonial (microeconómica), a fin de considerarlos a la luz de otras posturas que se revisarán en párrafos posteriores a este apartado.

Los autores postcoloniales, entre los que destacan, por un lado, Maloutas y Ghertner, por otro, Hamnet y MacFarlane, y Robinson, quienes se oponen al "difusionismo" de la teoría de la gentrificación como una imposición teórica que se difunde desde el norte al sur. Consideran que la gentrificación se circunscribe a un momento particular del norte global, específicamente, una fase post-industrial, la cual no corresponde con los procesos de desarrollo social y económico de Latinoamérica. Siguiendo lo anterior, afirman que para que se produzca la gentrificación debe siempre estar presente y como variable causal, cierto agente de alto capital económico y cultural como sujeto gentrificador, el cual es parte de esa fase post-industrial propia del capitalismo del norte.

Por todo lo anterior, se oponen a la validez interpretativa de la teoría como tal a cualquier otro momento, coyuntura o geografía fuera de ese contexto espacial y temporal propio del norte global (Betancur, 2014; Ghertner, 2015; Lees et ál., 2008; López, 2009; Maloutas, 2011). El presente trabajo se adscribe a la teoría de la oferta $y$ se coincide en que la gentrificación se trata, por lo general, de un proceso que guarda una relación muy estrecha con el funcionamiento del capitalismo, y como tal puede ser explicada (López, 2016). Siguiendo esta línea, se considera que es posible estudiar la gentrificación en sus particularidades para Latinoamérica y para el caso de Costa Rica dentro de un campo de estudios comparativos.

Si se retoman las tres fases de la gentrificación identificadas por Lees et ál. (2016) en su trabajo sobre lo que consideran una gentrificación planetaria, se podría afirmar que la gentrificación en Latinoamérica corresponde a la tercera fase: se presenta a partir de la década de los 90, tanto en ciudades del centro como en periferias mundiales, $y$ se caracteriza por su internacionalización con la participación de capital financiero mundial de mayor envergadura $y$ operando con mayor escala geográfica.

Lo anterior también coincide con autores que han venido estudiando procesos de cambio en la economía urbana de Latinoamérica y el mundo, relacionados con la gentrificación en la medida en que corresponden a procesos de privatización y desregulación que se iniciaron en los países de esta región, a partir del giro neoliberal y con los cambios en el estilo de desarrollo.

Concretamente, estos autores apuntan a la transnacionalización del mercado inmobiliario, así como, a la desregulación y la privatización de la gestión urbana, señalándolos como fenómenos globales intensificados en Latinoamérica en las últimas tres décadas y que son factores causales de la segregación residencial socioeconómica, los cambios en los patrones de la migración interna (hacia la migración intraurbana) y la gentrificación (Sassen, 1991; Borja y Castells 2002; Sennett, 2004).

La literatura explica cómo han ocurrido importantes transformaciones territoriales urbanas, producto de procesos de mundialización de la economía. Señala que en un contexto globalizado, el importante crecimiento del sector inmobiliario transnacional ha jugado un papel central en la extensión de nodos productivos y de comercio de las empresas, así como de una mayor inversión en bienes raíces, que apoyada en procesos de privatización y desregulación del desarrollo urbano, ha generado la fragmentación del territorio y la división social del espacio urbano (Janoschka, 2002; De Mattos, 2005). 
Asimismo, en los últimos años se ha pasado de un modelo de ciudad neoliberal a un modelo de ciudad empresarial, la que está más relacionada con la gentrificación por el estilo de gobernanza urbana que se promueve. En los primeros tres lustros del siglo XxI, la dinámica en Latinoamérica ha consistido en la reconquista de áreas centrales y pericentrales, en el período anterior, la aplicación de políticas neoliberales tuvo como efecto la expansión periférica (De Mattos, 2006).

La gestión urbana de tipo empresarial (con pocas regulaciones $y$ en donde el Estado participa del negocio inmobiliario directa o indirectamente) tiene su base principalmente en las asociaciones público-privadas, $y$ con escasos instrumentos de gestión; estas asociaciones operan bajo un esquema especulativo, es decir, basando sus operaciones en la obtención de ganancia (De Mattos, 2016 y Harvey, 1985). Mas allá de estos procesos de escala macro, conviene señalar algunas precisiones conceptuales y evidencias acerca de las manifestaciones de la gentrificación en Latinoamérica. A este respecto, Casgrain y Janoshka (2013) consideran que el estudio de la gentrificación en las ciudades latinoamericanas debe considerar al menos cuatro particularidades del contexto:

1) La constante creación de nuevos mercados inmobiliarios, esto es, la propagación de urbanizaciones cerradas en la periferia metropolitana. Procesos de urbanización suburbana con gran conflicto de clase: formas de invasión de terrenos $y$ autoconstrucción (fuera $o$ al margen de normativas del Estado), $y$ las consecuentes políticas urbanas neoliberales que restringen o desmantelan los asentamientos de autoconstrucción, a la vez que introducen reformas de liberalización del suelo y sistemas de subsidios (Casgrain y Janoshka, 2013, p. 26).

2) La gentrificación simbólica, que consiste en la restauración del patrimonio arquitectónico de los centros urbanos para turistas, elites locales y creciente clase media. Los programas de "rescate" del patrimonio suelen ser un pretexto para expropiar y con ello expulsar a la población pobre que ha ocupado históricamente el centro urbano. Además de otras consecuencias o efectos paralelos como expulsión de vendedores ambulantes para preparar el paisaje urbano para la posterior gentrificación (Casgrain y Janoshka, 2013, p. 26).

3) La particular debilidad en la gestión local, que se manifiesta a nivel macroeconómico en: privatización, apertura a la inversión extrajera, liberalización monetaria y desregulación del suelo urbano. Los instrumentos específicos de planificación neoliberal y liberalización de la construcción proporcionan una creciente acumulación de renta del suelo capitalizada por un pequeño grupo de grandes inversores. Por su parte las administraciones locales, carecen de recursos humanos y financieros para enfrentarse a los agentes privados, quienes controlan la tecnología, el suelo y el capital (Casgrain y Janoshka, 2013, p. 26).

4) Finalmente, quizá el rasgo mas particular es la fuerte presencia de luchas vecinales en contra de procesos actuales de reurbanización capitalista que presentan resistencia a la gentrificación. Estas protestas podrían desacelerar la gentrificación en América Latina a través de nuevos enfoques y experiencias populares (Casgrain y Janoshka, 2013, p. 26).

Sobre este último punto, es importante enlazarlo con lo afirmado por Díaz (2013), quien señala que en el caso de las ciudades latinoamericanas, conviene considerar un aspecto contingente, $y$ es el de los vecinos originales de los barrios gentrificados, cuyas características económicas y culturales particulares, constituyen un factor en el proceso de gentrificación:

...su carencia de capital cultural y social que impediría desarrollar estrategias efectivas de bloqueo frente a otros grupos $y / 0$ ser susceptibles a procesos de estigmatización por parte del conjunto de la sociedad. Esto se correspondería, en muchos casos, 
con una población mermada y envejecida, a veces lumpenproletarizada (Díaz, 2013, p. 20).

En cuanto a las diferentes manifestaciones y formas que asume la gentrificación en Latinoamérica, Salinas (2013) destaca con base en la revisión de una serie de trabajos empíricos, las siguientes tres formas de gentrificación como las más prevalentes: transformaciones en la imagen urbana, intervenciones en el patrimonio histórico e intervención privada en los centros históricos. Sobre la primera, destaca que en las ciudades latinoamericanas hay una fuerte tendencia de reforzamiento de los proyectos inmobiliarios con políticas de "limpieza" de rostro o imagen urbana, consisten en planes que tienen como objetivo mejorar la seguridad, el alumbrado y los espacios públicos, pero que implican la expulsión del comercio informal y la indigencia, tal y como se ha observado en Quito, Guayaquil y Ciudad de México (Salinas, 2013).

Sobre las intervenciones del patrimonio, Salinas (2013) señala que bajo el objetivo de recuperar el patrimonio histórico se emprenden proyectos asociados a un fuerte marketing urbano enfocado a la atracción del turismo, lo que hace a estos espacios más vulnerables a experimentar gentrificación. En la tercera de las manifestaciones, se destaca la intervención privada de los centros históricos que significa la liberalización y privatización de la gestión y el desarrollo urbano, asimismo, se observan políticas como la exención de pago de impuestos a la construcción, estímulos a la oferta y demanda, cambios en el uso del suelo o la ocupación del suelo.

Finalmente, sobre la gentrificación en la ciudad latinoamericana conviene referirse al tema del nuevo residente en áreas que se encuentran en este proceso, y para este efecto resulta revelador lo hallado por Contreras (2017), en su trabajo realizado en Santiago de Chile, en donde la autora identifica y describe al menos cinco tipos de sujetos inmigrantes al centro de Santiago en años recientes, a partir del Plan de Repoblamiento: "gentries pioneros y sucesores, residentes transitorios o "aves de paso", decadentes y precarios urbanos (estos últimos incluyen inmigrantes latinoamericanos)" (p. 117). La autora explica estas tipología como el producto de:

...la superposición simultánea o secuencial de procesos de renovación, inversión y gentrificación, con otros de tugurización, desinversión y deterioro, lo que provoca diferentes procesos sociales que coexisten $y$ por lo tanto exigen una nueva reinterpretación de los sujetos generadores del cambio (...) y del proceso de gentrificación como lineal y unidimensional (Contreras, 2017, p. 117).

\section{ASPECTOS HISTÓRICOS, GEOGRÁFICOS, SOCIOECONÓMICOS Y POLÍTICOS DE LA GAM}

Retomando el objetivo central de este trabajo, a saber, problematizar la forma en la que se debe abordar el estudio del fenómeno de la gentrificación, cómo interpretarlo y a partir de ello, plantear algunas interrogantes para una investigación posterior. A continuación se describe de manera sucinta el proceso histórico del desarrollo de las ciudades de la GAM y las políticas urbanas, a fin de plantear antecedentes que permitan señalar algunos elementos de orientación para aproximarse al estudio y comprensión de la gentrificación en el contexto costarricense.

Como primer punto, es importante señalar que en varios sentidos, el proceso de desarrollo de la ciudad es una manifestación de procesos generales de desarrollo nacional. La transformación del proyecto político y económico del país durante el siglo xx, que pasó de un modelo desarrollista con instituciones estatales que garantizaban un elevado acceso a sistemas de desarrollo social y empleo, a un modelo neoliberal en el cual se reduce la inversión pública y el tamaño del Estado, tuvo su correlato en las políticas urbanas, así como, en el crecimiento y desarrollo de la ciudad, su naturaleza, morfología y funcionamiento.

De esta forma, es posible identificar dos grandes períodos importantes en este proceso: 1949-1982 y 1982-2013. En el primer período, 
la tendencia fue la expansión urbana a través del cambio del suelo agrícola al uso urbano, se produjo una especie de colonización agrícola, con crecimiento industrial y un emergente sector urbanizador vinculado a las viejas oligarquías cafetaleras del Valle Central. Durante este período, el Estado emprendió una estrategia para ordenar el territorio y la ciudad, a través de la planificación y la regulación, la creación de instituciones relacionadas y la inversión en infraestructura pública.

En el segundo período, la expansión estuvo relacionada a un aumento del déficit habitacional de sectores bajos y medios que se organizaron $y$ tomaron terrenos en zonas no urbanizadas, así como, a un proyecto industrial transnacional y al desarrollo de proyectos urbanizadores de sectores inmobiliarios ya consolidados, dirigidos a sectores medios y altos en espacios antes rurales y áreas de conservación. Durante este período, el Estado realiza una serie de esfuerzos por impulsar planes de ordenamiento territorial y urbano, que a la vez desincentiva mediante la ausencia de inversión en infraestructura pública, la desregulación y cediendo el espacio al sector privado en la dotación de servicios, equipamientos e infraestructura urbana.

Retomando esta historia urbana, es posible señalar que para el año 1880, la ciudad capital San José tenía una población de 10000 habitantes, para entonces la planificación de la ciudad se limitaba al trazado de calles aplicando la cuadrícula española. Asimismo, en un intento por consolidar el carácter de la ciudad, "se construyeron cuatro iglesias en los cuatro costados del San José de entonces: Iglesia del El Carmen al norte; Iglesia La Dolorosa al sur; Iglesia La Merced al oeste; e Iglesia La Soledad al este" (Klotchkov, 2001, p. 4).

Hacia 1900, toda la población se localizaba en tres manzanas y es hasta 1910 cuando se desarrolla el primer barrio fuera del cuadrante: Barrio Amón. Seguidamente, entre 1920 y 1930, se desarrolla el segundo barrio residencial: Otoya. Desde esta década, el desarrollo del comercio en el centro expulsó a las clases medias y altas hacia un creciente cinturón de barrios residenciales: La Guaria, Los Yoses, La
Granja, Paseo Colón, Bello Horizonte, entre otros. Este cinturón se consolida en 1940 y prolifera de manera masiva a partir de 1950 (Láscaris, s.f.). Desde la mitad del siglo xIX y hasta mitad del siglo xx:

... el crecimiento de la mancha urbana es fuera de la zona compacta de la ciudad $y$ no tiene un patrón definido ya que se crece sin planificación, la ocupación del territorio y el uso urbano se desarrolla en función de las posibilidades de accesibilidad: primero hay crecimiento residencial de la zona rural y a medida que estos conglomerados se expanden, aparecen las urbanizaciones, las vías y los servicios (Klotchkov, 2001, p. 5).

Como resultado de este proceso, el crecimiento urbano se produjo a lo largo de las vías que comunicaban de forma radial a los pueblos periféricos del Valle Central con la ciudad capital. Durante este período, no se conoce de acciones para el desarrollo urbano desde la institucionalidad del país (Klotchkov, 2001).

Posteriormente, a la fundación de la Segunda República en 1948, se impulsó un modelo de Estado desarrollista que enfatizaba en la sustitución de importaciones y en generar procesos de planificación en todas las escalas territoriales $y$ dimensiones sectoriales. En este contexto, en 1948, se le encargó a Anatole Solow, miembro de la Unión Panamericana, la tarea de asesorar a las autoridades nacionales en la elaboración de una estrategia de planificación urbana para la ciudad capital San José (Solow, 1949).

El documento producido por Solow: Proyecto para el desarrollo urbano de la capital de Costa Rica (1949), se consideró como una reflexión con lineamientos técnicos de partida para la discusión en torno a la producción de proyectos de planificación urbana para San José. El documento identificó la problemática de la ciudad como el producto de haber seguido un patrón de crecimiento y concentración poblacional sin planificación y sin las herramientas institucionales, legales y técnicas necesarias. Solow (1949) llamó la atención sobre la urgencia 
de una planificación moderna orientada hacia el desarrollo racional $y$ funcional de la ciudad.

A partir de 1950, Costa Rica inicia una nueva etapa de crecimiento político e institucional bajo el modelo socialdemócrata y en este contexto, se crea el Instituto Nacional de Vivienda y Urbanismo (INVU) que asume la rectoría en materia de planificación urbana. Asimismo, se promulga la Ley nro. 4240 de Planificación Urbana (1968), primer instrumento legal propuesto para planificar el desarrollo de las ciudades del país y cuyos ejes principales giraban en torno a la distribución de la población, los usos de suelo y de la planificación del desarrollo de la producción en relación con el uso del suelo y los recursos públicos. Con esta misma ley se crea la Oficina de Planeamiento del Área Metropolitana de San José (art. 1, 63, 64 y 65).

En 1973, se produce el documento técnico titulado Regionalización de Costa Rica para la planificación del desarrollo y la administración, primer informe en establecer una propuesta de regionalización territorial para el territorio costarricense, el cual estuvo a cargo del geógrafo alemán Helmut Nuhn. En 1976, se inicia la elaboración del Plan DRENACA, cuyo fin era el control de los escurrimientos pluviales, la protección de las aguas subterráneas y problemas ambientales relacionados. Se considera una propuesta muy completa en términos urbanos, pues constituye un plan maestro para el ordenamiento del área central de San José, entre otras cosas, analizaba opciones de desarrollo urbanístico como la ciudad central, la ciudad paralela y el uso del suelo mixto (Municipalidad de San José, 2017).

A partir de este momento, el "tema urbano" se torna de interés, integrandose en el lenguaje y el actuar institucional. Empieza a pensarse en la importancia de contar con la delimitación de una región metropolitana, en la que se articulen las dinámicas económicas, sociales y políticas del área central del país. A partir de la década de 1960, se aceleró la expansión de la mancha urbana, por una parte potenciada por el desarrollo de la industria, por otra parte, por un naciente mercado inmobiliario. San José se consolida como el nodo urbano principal que articula una dinámica regional, pero en constante crecimiento, $y$ es así como se empieza a formar una espacio metropolitano, con la conurbación de las tres cabeceras de las provincias centrales: San José, Heredia y Alajuela (Carvajal y Vargas, 1987).

La metropolitización de San José fue el resultado de un proceso histórico en el que convergen actores tanto públicos como privados, pero sobre todo de la dirección de un Estado que desarrolló una estrategia para la obtención de los beneficios económicos que traería una renta urbana para el proceso de acumulación de capital. Este fue un proceso en la región del país que tenía una mayor trayectoria capitalista, explotando la concentración de población, la infraestructura de comunicación y la concentración de servicios básicos (Carvajal y Vargas, 1987). Algunos de los proyectos de urbanización desarrollados por el Estado en el período fueron Los Hatillos, como modelo de ciudad satélite, la construcción de la autopista General Cañas y el Aeropuerto Juan Santa María.

Pese a los esfuerzos mencionados por planificar el desarrollo del territorio de la ciudad capital, en el período que va del año 1949 y hasta inicios de la década de 1980, las acciones institucionales son débiles a nivel metropolitano y se estimula la urbanización sin contar con bases sólidas para la planificación metropolitana. Esto debido a que si bien existieron estudios técnicos, la acción gubernamental sobre esas bases fue más de una reflexión acerca del curso que debía tomar el crecimiento de la ciudad y menos de unas acciones articuladas, sobre todo en lo que concierne a regular la acción del sector privado (Brenes y Molina, 2013).

Ante la evidencia en la ciudad de que el crecimiento de la mancha urbana desde la década de 1950, había sido débilmente planificado, y se avisoraba un funcionamiento caótico de la ciudad (Brenes y Molina, 2013). En la década de 1980, surge la iniciativa de contar con una herramienta para distribuir la población en las áreas prioritarias y de acuerdo con las densidades más factibles (INvU, 1983), es así como se elabora en 1982, el primer proyecto de planificación urbana de escala regional, el Plan 
Regional Metropolitano Gam 1982 (Plan GAM 1982). Con el Plan GAM 1982, surge la Gran Área Metropolitana (GAM), apareciendo por primera vez delimitada como conglomerado urbano metropolitano a partir de estudios técnicos que definen este territorio por sus características geográficas, económicas y sociales, además de aspectos determinantes del relieve (MIVAH y Cooperación Financiera de la Unión Europea, 2009).

La GAM corresponde muy cercanamente con la delimitación del Valle Central, por lo que es un territorio intermontano; su área es de $1997 \mathrm{~km}^{2}$, que equivale al 4\% del territorio nacional, y para 1984 albergaba 1326 443 habitantes, es decir, cerca del $50 \%$ de la población nacional. De acuerdo con la división político-administrativa, incluye porciones de las cuatro provincias centrales y suma 31 cantones (MIVAH y Cooperación Financiera de la Unión Europea, 2009).

Este conglomerado urbano, concebido así en el Plan GAM 1982, tiene la particularidad de contener una amplia zona rural en el borde $y$ la periferia, aunque se considere como un todo un conglomerado urbano o área metropolitana. De esta forma, en este plan se establecieron tres anillos para planificar su crecimiento: el anillo central o anillo de contención urbana, el segundo anillo destinado a la producción agropecuaria y el tercer anillo reservado para la conservación ambiental (MIVAH, 2009). Para el año 2007, la GAM concentraba el 54\% de la población del país y en ella se producía el $80 \%$ del Producto Interno Bruto (PIB) (MIVAH y Cooperación Financiera de la Unión Europea, 2009).

Ahora bien, al igual que en otros países latinoamericanos, a partir de la década de 1980 se sucedieron en el país una serie de políticas de privatización y reconfiguración de la gestión urbana a nivel regional (GAM) (Mora y Solano, 1993; Klotchkov, 2001 y Pérez, 2005), que cambiaron el patrón de crecimiento y el rol del Estado y del sector privado en el desarrollo urbano. Justamente, el recién aprobado Plan GAM de 1982 fue muy debilitado por estas políticas que marcan el inicio del retiro del Estado en materia urbana.
La crisis económica, la que se ha dado en llamar la "década perdida" de los años 80, significó para el país un incremento de casi 10\% en el porcentaje de pobreza. El modelo desarrollista implementado desde la constitución de la Segunda República se vio fuertemente impactado por la caída en el mercado de los precios del café y el banano, productos que generaban gran parte del ingreso fiscal del país (Seligson y Muller, 2012).

Esta crisis afectó fuertemente la capacidad adquisitiva de los salarios en rubros específicos (entre ellos, el de vivienda). Se estima que la reducción del índice de accesibilidad de la población a la vivienda fue de un 50\% entre 1980 y 1985 (Gutiérrez y Vargas, 1997, citado por Pérez, 2005). La carencia habitacional provocó la emergencia de un gran número de comités de lucha por la vivienda, los cuales llegaron a aglutinar cerca del $10 \%$ de la población de la GAM, y organizaron la ocupación informal de terrenos de grandes dimensiones (Valverde, 1990, citado por Pérez, 2005; Molina, 1990).

Ante este panorama, se hizo una declaración de emergencia nacional sobre la problemática del déficit habitacional. Esta declaratoria promovió un estado de excepción en la legislación urbana vigente a fin de tomar medidas de emergencia para atender el déficit habitacional, la primera de estas medidas fue suspender la aplicación del Plan GAM de 1982, principalmente en lo que se refería al anillo de contención urbana, es decir, se permitió construir fuera del anillo y en áreas de desarrollo agrícola y protección ambiental (Mora, 2003; Pérez, 2012).

Para atender "la emergencia" se estableció desde 1983 la Comisión Especial de Vivienda (CEv, instancia adscrita a la Comisión Nacional de Emergencias pero que obedecía directrices del Ministerio de Vivienda y Asentamientos Humanos (міvAH). Bajo la dirección de esta Comisión, en general, los procesos de reubicación o de regularización de asentamientos precarios se hicieron con "mucha flexibilidad", es decir, sin respetar la legislación vigente y bajo la gestión de empresas inmobiliarias privadas, desde aspectos operativos del sistema financiero hasta actividades de construcción, siendo hasta ese 
momento, el tema de la vivienda de interés social exclusivo del Estado (Román, 2013).

En 1986, se institucionalizaron estas prácticas con el impulso de la reforma legal del Sector Estatal de Vivienda y Asentamientos Humanos, y se promulgó la Ley del Sistema Financiero Nacional para la Vivienda (SFNV). Con esta reforma, se migró a un modelo público-privado en el cual el Estado financia la demanda de vivienda mediante bonos, pero estos fondos son administrados por la banca privada $y$ son las empresas inmobiliarias privadas las que se encargan del proceso constructivo de acuerdo a la demanda (BANHVI, 2018; Pérez, 1998).

Como parte de este proceso de privatización de la gestión urbana en el tema de la vivienda, durante la década de los años 1980, se inicia una etapa de cambio en el estilo de desarrollo económico y social del país, se da un viraje neoliberal y la reducción del Estado minimiza la capacidad de inversión pública y en general, las instituciones relacionadas con el urbanismo no invierten en los proyectos del Plan GAM 1982 (Klotchkov, 2001). De manera que, desde entonces se ha continuado bajo un esquema legal en la planificación urbana (Ley de Planificación Urbana 4240, particularmente, el Plan Gam de 1982), que fue concebido bajo un modelo de planificación desde el Estado, sin embargo, en un escenario de Estado disminuido, desfinanciado, que es más ausente que ejecutor y menos regulador, pues en la Ley 4240 , las regulaciones a la acción privada en el territorio son débiles en tanto el espíritu de la Ley obedece a un concepto de Estado que se encarga de toda la planificación, el financiamiento $y$ la ejecución (benefactor).

Con el retiro paulatino del Estado en la inversión y el desarrollo urbano general, emergen necesidades urbanas sin satisfacer $y$ vacíos, con lo cual se crean nichos de mercado para las empresas privadas (vivienda, infraestructura, zonas verdes, centros recreativos, malls, etc.), las que actúan bajo regulaciones debilitadas 0 violentadas (Brenes y Molina, 2013). Desde entonces se produce un crecimiento caótico de la ciudad como producto de un modelo inacabado de ciudad compacta y de un crecimiento expansivo no planificado y con pobre regulación
(MIVAH, 2009) que da lugar a una ciudad más bien fragmentada (Janoschka, 2002).

Se han planteado diversas propuestas para actualizar el Plan GAM de 1982 y modificar el esquema de gestión urbana hacia uno de tipo empresarial que se base en alianzas públicoprivadas $y$ otras herramientas de gestión mixta $y$ figuras legales que otorguen mejores condiciones para la acción del sector privado. Asimismo, se ha planteado la necesidad de contar con este marco legal $y$ administrativo para poder avanzar planes y proyectos de renovación urbana $y$ repoblamiento, pues en el esquema actual no son viables, sin embargo, estas propuestas no han sido aprobadas.

A continuación, se resumen los elementos centrales de estas propuestas a fin de comprender tanto el concepto de ciudad como el de repoblamiento y renovación urbana, incluido su esquema de gestión urbana, que no pudiendo ser ejecutadas, dibujan la naturaleza de lo que podría ser el esquema de la renovación y revitalización. Si bien, esto no se ha dado, constituye el escenario en el que podría estarse dando la gentrificación actualmente, es decir, ante la ausencia de herramientas para su desarrollo en las condiciones en las que se presenta en otras ciudades latinoamericanas.

En el Plan GAM de 1982, aparece la primera propuesta que se podría denominar moderna $y$ de escala regional, de regeneración y renovación urbana - el tema se discutía desde décadas atrás, por ejemplo, en el primer Proyecto Ciudad Gobierno- que resalta la idea del repoblamiento y la renovación urbana, en el cual bajo un modelo de ciudad compacta se plantean mayores densidades en el área central de la GAM $y$ se señalan las áreas que deben ser renovadas $y$ densificadas de manera prioritaria. El Plan indicaba conglomerados de tugurios y zonas de vivienda deteriorada en un mapa base que orientaba acciones posteriores. Estos se clasificaron en tugurios circunscritos o continuos, diseminados y localizados, los que albergaban a 73000 habitantes en un área correspondiente al 14\% de la superficie de la GAM (INvU, 1983). Sin embargo, como se explicó párrafos atrás, esta propuesta no llegó a ejecutarse, pues en los años posteriores a la aprobación del Plan, 
se priorizó el desarrollo de vivienda de interés social nueva en la periferia de la ciudad.

La segunda propuesta se encuentra contenida en la Fase II del Plan Nacional de Desarrollo Urbano (Consejo Nacional de Planificación Urbana-CNPU, 2002), plantea un Sistema de Áreas de Regeneración que define catorce áreas de la ciudad de San José (Pitahaya, San Cayetano, Hatillos, Don Bosco, Amón, La California, entre otros); propone tratamientos urbanos $y$ herramientas legales $y$ financieras para el establecimiento de alianzas público-privadas que ejecutarían el plan (CNPU, 2002).

También, en esta fase del Plan Nacional de Desarrollo Urbano, se inicia la creación de la Comisión de Repoblamiento de San José, conformada por la Municipalidad de San José, el Consejo Nacional de Planificación Urbana, Invu, MIVAH, Cámara de la Construcción, Consejo Inmobiliario, algunas otras instituciones autónomas, entre otros. La Comisión de Repoblamiento tenía como objetivo crear de manera concertada entre sectores, un reglamento que hiciera posible la regeneración y renovación urbana de San José con el objetivo de densificar y repoblar. Este proceso de diálogo aún no ha acabado y el marco legal aún no es una realidad (Brenes y Molina, 2013).

Por otro lado, el Plan Regional Urbano de la Gran Área Metropolitana (PRUGAM 20082030), propuesta de actualización del Plan GAM de 1982, propuso la figura legal para hacer posible la renovación urbana por parte de $\mathrm{Mu}$ nicipios vía Planes Reguladores, introdujo la herramienta del plan parcial de renovación urbana, dentro de la figura de planes especiales (MIVAH, 2009). Luego de PRUGAM, que no fue aprobado, se han sucedido dos propuestas de planificación urbana para actualizar el Plan GAM de 1982: POTGAM y Plan GAM 2013, las que tampoco fueron aprobadas.

Finalmente, la Municipalidad de San José desde el año 2005 (PDUSJ, 2015), emprendió un Plan de Repoblamiento para el casco central. El plan no incluye proyectos de renovación urbana como tal, a ejecutar por el Estado o en alianza con el sector privado, el planteamiento consiste en modificaciones al Plan Director Urbano de San José que liberan al alturas y densidades mínimas permitidas, por otra parte, no brinda otras herramientas como subsidios a la oferta o la demanda, ni las figuras legales para operar en un esquema diferente al de la legislación actual, por ejemplo, en materia de renovación de edificios desocupados-abandonados, zonas deterioradas, etc.

El pretendido repoblamiento de San José no presenta resultados contundentes, pues los datos demográficos siguen mostrando que la población no aumenta de manera sostenida. Según datos de los últimos censos (1984-20002011) la población del cantón de San José viene disminuyendo, pasando de 309672 en 1984, a 288054 en 2000, y a 241464 en 2011, y su Saldo Neto Migratorio, el segundo más alto en el país de signo negativo es de -14.4 en el año 2011 (Barquero y Molina, 2014). De igual forma, si se consideran solamente los cuatro distritos centrales, la población de la ciudad de San José alcanzó 56000 personas en el año 2000, cifra cercana a la de la población registrada en el censo de 1927 (Rosero, 2002); y según el Censo de 2011, solamente 23399 habitantes han llegado a vivir en El Carmen, Hospital, Catedral, Merced y Mata Redonda (Brenes y Molina, 2013). A la luz de estos datos, inquieta conocer las características y dimensiones de la gentrificación, pues de darse, sería en un contexto de despoblamiento, con un número limitado de inmigrantes o vecinos nuevos, al menos en lo que se refiere al casco central de San José.

\section{DISCUSIÓN}

Para el abordaje del caso costarricense, se considera inviable utilizar el enfoque postcolonial que rechaza la gentrificación por considerarla una narrativa proveniente del norte global inaplicable al sur global.

Siguiendo la literatura consultada, la que se expuso en el primer apartado de este trabajo, se considera adecuado comprender la gentrificación como un fenómeno planetario que ha evolucionado en el tiempo, que se presenta en múltiples manifestaciones y que en su tercera fase, a partir de la década de los 90, se presenta tanto en ciudades del centro como en periferias mundiales, incluida Latinoamérica. Asimismo, se considera que la gentrificación forma parte 
de un proceso de avance neoliberal, intensificado en Latinoamérica en las últimas tres décadas, que apunta a la transnacionalización del mercado inmobiliario, así como, a la desregulación y privatización de la gestión urbana; todos procesos que se verifican en nuestro país a partir de la década de 1980, tal y como se expuso en el apartado anterior.

Para estudiar el caso costarricense, conviene utilizar el supracitado enfoque macro, que explica la gentrificación con base en el concepto de brecha de renta, entendiéndola desde las estructuras capitalistas que la generan pero considerando particularidades sociológicas, culturales e institucionales que el fenómeno adquiere en cada lugar. En este sentido, el capítulo anterior recoge las particularidades de este contexto y explica la historia política, socioeconómica y espacial de la ciudad. Justamente, porque se ha sostenido que la gentrificación es un proceso en el tiempo y el espacio, y que no es el artefacto (la construcción en sí) sino los procesos económicos, urbanos, comerciales y culturales que dan lugar a la generación, captura y acumulación de la brecha de renta en contextos más amplios como la escala del barrio o la ciudad.

Ahora bien, siguiendo los puntos anteriores $y$ lo reportado en amplia bibliografía sobre estudios de caso comparados en Latinoamérica (Casgrain y Janoshka, 2013; Contreras, 2017; López, 2013; Matus, 2017; Rojo, 2016; Salinas, 2013), hay una característica común en los diferentes procesos experimentados por las ciudades: políticas urbanas que de forma directa o indirecta facilitan o promueven la gentrificación, o mejor dicho, la captura y acumulación de la renta urbana y la consecuente expulsión de población. Tal y como explica Salinas (2013), son comunes los planes de repoblamiento, los procesos de rescate del patrimonio histórico y los planes de renovación o regeneración urbana como los instrumentos generadores de procesos de gentrificación. Aunque la renovación urbana como tal no debe equipararse a la gentrificación (Díaz, 2013), obedece a políticas urbanas y se basa en una serie de instrumentos de gestión urbana que permiten, entre otros, las alianzas público-privadas, la desregulación y el financiamiento público para que sea posible que el mercado inmobiliario se consolide como un actor central del proceso.

En el caso de Costa Rica, los planes de densificación, repoblamiento y renovación de la capital $-y$ los centros urbanos de la GAM o de ciudades secundarias-, aún están en proceso, como se explicó en el apartado anterior, la Comisión de Repoblamiento de San José trabaja en la gestión y el diálogo entre sectores para concertar $y$ diseñar acciones. El Reglamento de Renovación Urbana (RRU) (Reglamento 6259), se aprobó recientemente en el primer semestre del año 2018, con lo cual, a la fecha se cuenta con escasas herramientas concretas de gestión urbana, por ejemplo, se tienen las herramientas que ya contemplaba la Ley 4240 , pero estas fueron diseñadas para un contexto de Estado benefactor, no plantean un esquema de gobernanza urbana empresarial, el cual haría más expeditas $y$ agresivas las acciones del sector privado $y$ del público, también en un papel de gestor, inversionista, socio y financista, quizá por eso aún no se ven resultados claros en la ciudad de un proceso de repoblamiento o renovación urbana (Brenes y Molina, 2013).

Ante este panorama, surge una primera interrogante ¿cómo se han dado los procesos de producción y captura de brecha de renta $y$ los de expulsión de población y en qué áreas de la ciudad?, si es que estos se dan, entonces ¿cuál es la particularidad de la gentrificación en Costa Rica si se la compara con lo hallado en otras ciudades latinoamericanas? En este sentido, se hace necesario analizar el esquema de gestión urbana que tiene el país, $y$ detenerse en al menos dos puntos importantes, que constituyen la base de la tesis central de este trabajo, la cual consiste en que el esquema de gobernanza urbana no favorece la gentrificación.

Como primer punto, la ausencia de renovación urbana: la falta de instrumentos legales y financieros para la renovación urbana, el repoblamiento, la rehabilitación, entre otros, propios de un modelo de urbanismo empresarial. En Costa Rica, por ejemplo, no existe una normativa favorable a la rehabilitación de edificios deteriorados o abandonados. Asimismo, no hay municipios invirtiendo en 
proyectos residenciales, ni financiando al desarrollador o al comprador.

En el caso de la Municipalidad de San José, ha implementado incentivos al desarrollo vertical liberalizando las alturas y densidades, pero la gestión y el financiamiento son responsabilidad exclusiva del empresario inmobiliario. Por estas razones, como lo mencionado anteriormente, en el caso costarricense se puede hablar de un Estado o municipios que actúan mas por omisión y desregulación. De esta manera, el parque habitacional y los edificios de los centros urbanos y particularmente, del casco central de San José, permanecen intactos $y$ deteriorados desde hace décadas.

Siguiendo lo anterior, como segundo punto, se puede afirmar que existe un urbanismo neoliberal y modelo expansivo de ciudad. Podría afirmarse que Costa Rica se encuentra "en un modelo anterior" de expansión de la ciudad, como le llama De Mattos (2016), es decir, que si en los primeros tres lustros del siglo xxi, la dinámica en Latinoamérica ha consistido en la reconquista de áreas centrales y pericentrales, en el período anterior, la aplicación de políticas neoliberales tuvo como efecto la expansión periférica; es decir, que si en los primeros tres lustros del siglo XXI, la dinámica en Latinoamérica ha consistido en la reconquista de áreas centrales y pericentrales, en el período anterior, la aplicación de políticas neoliberales tuvo como efecto la expansión periférica. Esta tendencia se observa en metrópolis de mayor dimensión, $y$ también en algunas menores como La Paz, Montevideo, Ciudad de Panamá y San José de Costa Rica, entre otras (De Mattos, 2016).

Por estas razones, en el caso costarricense se habla de un modelo desregulado, neoliberal, y menos de un modelo empresarial en donde gobierno o municipios invierten $y$ participan como desarrolladores. La falta de herramientas legales $y$ financieras que apoyen $y$ financien a la empresa inmobiliaria, así como que favorezcan las alianzas público-privadas, han desincentivado la inversión y la renovación en las áreas centrales, concentrándola en la periferia urbana, en zonas rurales a las que se les cambia el uso del suelo de rural a urbano, y de esta forma el proceso de su urbanización ocurre con amplios márgenes de ganancia.

El escenario expuesto es el de una gestión urbana neoliberal, no empresarial tal y como se observa en otras ciudades latinoamericanas. Se tiene un Estado ausente que actúa más por omisión, que no financia o ejecuta proyectos, ni facilita los proyectos del sector privado. El caso de Costa Rica muestra lo que se podría denominar un "atraso" de las políticas urbanas necesarias para gestionar y acelerar la gentrificación, sin que esto signifique que no haya procesos de urbanización privada que posiblemente estén gentrificando, aunque sea vía operaciones especulativas a falta de apoyo financiero y administrativo; lo que supone que algunas modalidades de urbanización sean inviables por ser engorrosas y no rentables para las empresas inmobiliarias, como por ejemplo, la renovación de edificios desocupados-abandonados, en cuyo caso queda la opción del redesarrollo y la verticalización. Todos estos elementos permiten problematizar $y$ hacerse preguntas acerca de las condiciones en las que los factores $y$ procesos macro (y micro) de la gentrificación podrían estar actuando en el territorio.

Ahora bien, resulta interesante considerar algunos datos de estudios técnicos o investigaciones sobre movilidad residencial y otros fenómenos urbanos, que dan pistas sobre los patrones de la migración interna que podrían estar asociados a procesos de gentrificación. Por ejemplo, estudios del MIVAH señalan la movilidad de población de estrato medio y medio bajo hacia zonas pobres como el fenómeno de las cuarterías (MIVAH, 2015), la llegada de inmigrantes internacionales de estrato medio hacia viejas urbanizaciones de vivienda de interés social que ya están en proceso de despoblamiento (MIVAH, 2015). Con lo cual surge la pregunta, ¿podría verificarse en este proceso una especie de "filtrado hacia abajo"?, —siguiendo el término de Díaz (2013) que se explicó en el primer apartado-.

Por otra parte, el estudio de la migración intraurbana en la GAM señala la inmigración masiva de población de ingresos medios y altos hacia zonas de transición rural urbana (Molina, 
2009); $y$ también se presentan amplias zonas en la periferia que por características como menor dinámica económica y menor oferta habitacional, así como, envejecimiento moderado de la población, están constituidas en su mayoría por población estabilizada no migrante, es decir, que no expulsan ni atraen población (Molina, 2009), ¿habrá en unas y otras de estas zonas condiciones favorables o desfavorables para la gentrificación?

En estas investigaciones, se explica que el estudio detallado de los datos de la migración interna en la GAM, entre los años censales 1984 y 2000, muestra que los mayores flujos migratorios van en su mayoría del centro al centro, es decir, los cantones con altas tasas de inmigración y emigración, son los mismos cantones del área central de la GAM: Montes de Oca, Tibás, San José, Goicoechea, Moravia, Heredia, Curridabat, Escazú, San Pablo, Flores, Vásquez de Coronado, Alajuelita y Santo Domingo, entre otros (Molina, 2009). Para el censo del año 2011, la tendencia es del centro al oeste y del centro al noroeste, aunque el flujo de dirección centro-centro sigue siendo importante numéricamente (Barquero y Molina, 2014).

Por otra parte, de forma intuitiva y con algún conocimiento general de la GAM - por supuesto a la espera de resultados-, y con el apoyo de algunos estudios relacionados, es posible reflexionar acerca de posibles formas de gentrificación en la GAM; por ejemplo, no se observan indicadores "a simple vista", de que se presente la llamada "slum gentrification", ya que los 182 asentamientos informales de la GAM, permanecen tal cual y no se conoce de proyectos urbanísticos sobre los mismos, más bien una serie de regulaciones impiden su urbanización, incluso para erradicarlos en sitio, pues la mayoría está en zonas de riesgo ambiental (MIVAH, 2009).

Asimismo, se observa en los últimos 15 años, el crecimiento urbanístico muy acelerado de los cantones de Escazú y Santa Ana con el desarrollo de condominios cerrados y torres de apartamentos dirigidos a un estrato socioeconómico alto, con la consecuente inmigración de extranjeros de origen venezolano y estadounidense en zonas exclusivas; y cuyo desarrollo ha significado el cambio de uso del suelo de rural a urbano y prácticamente, la extinción de las antiguas zonas agropecuarias y los poblados tradicionales (Molina, 2013). En años aún más recientes, se observa una urbanización acelerada con la consecuente oferta de vivienda en diversas zonas de Cartago.

Respecto a que la renovación urbana en las áreas centrales pareciera no contar con condiciones favorables, ¿será posible que la captura de brecha de renta se presenta en zonas rurales de la GAM?, ¿será posible que en la especulación causada por el cambio en el uso de suelo de rural a urbano, hay una condición más favorable para capturar y acumular brecha de renta? Quizá en la urbanización masiva de las zonas rurales bajo la figura del condominio cerrado $y$ las "quintas" o pequeñas propiedades que se urbanizan dentro de la normativa de parcela agrícola, esté la respuesta.

En cuanto a las áreas centrales de la ciudad, se observan algunas dinámicas que llaman la atención: barrios como Escalante y Aranjuez presentan fuerte desarrollo comercial y verticalización; Barrio Luján con gran deterioro y abandono producto de inundaciones, ocupándose con torres que se erigen sobre las otrora viviendas deterioradas o abandonadas; Barrio Los Yoses, con avanzado envejecimiento $y$ despoblamiento, ocupándose con población joven de clase media y gran desarrollo de emprendimientos de arte y diseño, etc. (моРт, 2015). En el caso de Los Yoses, recién hay compras de terrenos y la primera torre de apartamentos y oficinas será construida en abril de 2019, pero el barrio ya está posicionado como "barrio de moda" y sufre una gran presión por compra de viviendas y terrenos (Asociación de Vecinos de Los Yoses, 2017).

Lo que se ha observado en la ciudad es que, aparentemente, ante la ausencia de condiciones para la renovación urbana, hay procesos de verticalización y redesarrollo, no en áreas centrales, en el centro histórico o los barrios tradicionales de San José, sino que es en barrios del pericentro en donde el mayor tamaño de las propiedades permite al empresario inmobiliario una gestión más rápida que la que implicaría la negociación de compra-venta con un gran número de propietarios en un escenario de poco 
acompañamiento $y$ base legal para proceder de forma más expedita, por ejemplo, con una normativa más agresiva de expropiación con declaratoria de renovación o patrimonio. Llama la atención la verticalización en barrios de clase media $y$ alta del pericentro: Sabana, Escalante, Los Yoses y Dent. A este respecto, López (2014) señala esta realidad para Santiago de Chile: el modelo chileno de propiedad privada del suelo urbano es de una estructura desconcentrada, en donde la característica principal es la minúscula propiedad del suelo.

Por otra parte, el Proyecto "Ciudad Gobierno. Eje de Renovación Pacífico-Plaza Víquez", financiado por el Banco Interamericano de Desarrollo (BID, 2017), en elaboración actualmente, promueve un agresivo plan de renovación urbana en los barrios antiguos de Los Ángeles, La Dolorosa, La Soledad, La Cruz, San Cayetano y Cristo Rey; sin embargo, está en proceso la gestión que daría lugar a su ejecución.

En este punto, es central recordar que el Reglamento de Renovación Urbana (RRU), nro. 6259, se aprobó en el primer semestre del año 2018, y habrá que esperar a ver sus efectos una vez se aplique. Por lo pronto, especialistas en planificación urbana consideran que aún tiene debilidades en cuanto a mecanismos e instrumentos de gobernanza urbana de tipo empresarial, y sigue manteniéndose en un esquema basado en la desregulación (Arce y Chavarría, comunicación personal, 07/12/2018).

Ciertamente, el Reglamento nro. 6259 indica que en los próximos 4 años posteriores a la aprobación de este será el sector privado quien proponga las zonas y proyectos para la renovación, que hasta pasado ese período, serán los municipios quienes deban proponer sus propias zonas de renovación urbana, integrarlas en el Plan Regulador y elaborar un plan de ejecución y financiamiento.

A este respecto, el Consejo de Desarrollo Inmobiliario (CODI) opina que este reglamento es una buena promesa para el desarrollo inmobiliario en los centros urbanos, que tiene algunas debilidades pero también le da un gran espacio de acción al inversionista (CODI, comunicación personal, 07/12/2018).
En una investigación realizada en Santiago de Chile (López, 2013), se encontró que existen procesos deliberados de devaluación de renta de suelo socialmente capturada ¿Se estará provocando también en la GAM procesos deliberados de devaluación?, ¿la lentitud en la aprobación del Reglamento de Renovación Urbana habrá jugando este papel?, ¿existirá además de captura y acumulación de renta urbana, monopolio sobre esa renta? En esta misma investigación, se encontró evidencia de incrementos de renta potencial monopólicamente capturados, de ahí que interesaría conocer el número de inmobiliarias implicadas y sus operaciones en Costa Rica, por ejemplo, se conoce que ciertas empresas inmobiliarias son las principales desarrolladoras de toda la zona de Heredia, no solamente por ser propietarias de una gran cantidad de terrenos, así como grandes compradoras de terrenos otrora cafetales, sino porque además son las inversionistas y constructoras. Por otra parte, se conoce de ciertas empresas desarrolladoras o agencias inmobiliarias que controlan un amplio porcentaje de las operaciones de compra $y$ venta de propiedades en la GAM.

Todo lo examinado hasta este punto abre preguntas acerca de si se está presentando gentrificación en la GAM o el casco central de San José, y de ser así, resulta inquietante conocer cuáles son las particularidades y características que presenta en el contexto nacional, y si será posible identificar tipologías de desarrollo urbano y zonas de la ciudad bajo las que se presenta. Asimismo, ¿cuál es la magnitud del fenómeno? y ¿cuáles son las características de los procesos de captura y acumulación de renta urbana?, así como, la importancia en cifras de los movimientos migratorios y la expulsión de población, además, ¿cuáles serán las características socioeconómicas de los migrantes de entrada $y$ salida?

Se debe recordar que los principales investigadores de la gentrificación en Latinoamérica, utilizan conceptos de gentrificación que la definen a partir de las siguientes características coincidentes: la reestructuración espacial de un área urbana, mediante la reinversión de capital en mercado inmobiliario e infraestructura, con 
el mejoramiento de condiciones sociales por grupos de mayores ingresos que los residentes tradicionales o históricos, que provoca cambios en el paisaje urbano y el desplazamiento directo o indirecto de población, en un contexto de mercantilización del suelo (Janoschka, 2016; López, 2013; Salinas, 2013). Siguiendo el último párrafo, de presentarse la gentrificación en Costa Rica, surge la pregunta, ¿podría entenderse desde este concepto, o tendrá particularidades propias?, sobre todo si se considera que al parecer no hay renovación urbana en el casco central de San José, sino redesarrollo y verticalización. Asimismo, ¿se le podría llamar gentrificación a la urbanización de las zonas rurales?

Finalmente, retomando nuestro marco teórico, es importante llegar a comprender la tensión entre Estado, mercado y habitantes/pequeños propietarios (potenciales desplazados), en un proceso de transformación de un barrio o zona; comprender un proceso en el que se determine actores, acciones detonantes, momentos clave, entre otros. Comprender la tensión que existe entre sujeto gentrificador (sector privado/empresa inmobiliaria) y pequeño propietario, asimismo, la tensión entre sector privado/empresa inmobiliaria y gobierno local o instituciones del Estado del sector (INVU, MIVAH, municipios). Por último, comprender la acción de los vecinos y su dirección hacia el mercado y/o el Estado. En ese proceso, de manera transversal se debe comprender cómo la normativa $y$ gobernanza urbana facilita o bloquea la transformación urbana que lleva a un proceso de gentrificación.

\section{REFERENCIAS}

Banco Interamericano de Desarrollo-BID. (2017). Regeneración urbana de los Cuatro Distritos Centrales de San José. Recuperado de https://www.iadb.org/es/ project/CR-T1160

Banco Nacional Hipotecario para la ViviendaBAnHvi. (2018). Ley del Sistema Financiero Nacional para la Vivienda nro. 7052. Recuperado de http://www.banhvi. fi.cr/quienes_somos/leyes_reglamentos/ Ley7052_Dic17.pdf
Barquero, J. y Molina, W. (2014). Migración Intraurbana. Hacia un Nuevo Patrón de la Migración Interna en Costa Rica. Instituto Nacional de Estadística y Censos (INEC)-Costa Rica a la luz del Censo 2011. San José, Costa Rica.

Betancur, John. (2014). Gentrification in Latin America: Overview and Critical Analysis. Urban Studies Research, 1-14.

Brenes, E y Molina. W. (2013). Regeneración y repoblamiento de San José aún no llegan. Revista Ambientico, (234), 15-25.

Borja, J. y Castells, M. (2002). Local y global. La gestión de las ciudades en la era de la información. Madrid: Editorial Taurus.

Carvajal, Guillermo y Jorge, Vargas. (1987). El surgimiento de un espacio urbano metropolitano en el valle central de Costa Rica. 1950-1980. Anuario de Estudios Centroamericanos, (13).

Casgrain, A y Janoschka, M. (2013). Gentrificación y resistencia en las ciudades latinoamericanas. El ejemplo de Santiago de Chile. Andamios. Revista de Investigación Social, 10 (22), 19-44.

Concejo Nacional de Planificación UrbanaCNPU. (2002). Plan Nacional de Desarrollo Urbano. Gobierno de la República, San José, Costa Rica.

Gobierno de la República de Costa Rica. (1968). Ley nro. 4240 de Planificación Urbana. San José, Costa Rica.

Contreras, Y. (2011). La recuperación urbana y residencial del centro de Santiago: nuevos habitantes, cambios socioespaciales significativos. Eure, 37 (112), 89-113.

Contreras, Y. (2017). De los "gentries" a los precarios urbanos. Los nuevos residentes del centro de Santiago. Eure, 43 (29), 115-141.

De Mattos, C. (2005). Gestión territorial y urbana: de la planeación a la governance. Ciudades, (66), 2-9.

De Mattos, C. (2006). Modernización capitalista y transformación metropolitana en América Latina: cinco tendencias constitutivas. En Geraiges de Lemos, A. I., Arroyo, M. y Silveira, Ma. L. (Eds). América Latina: cidade, campo e turismo. San Pablo: 
Consejo Latinoamericano de Ciencias Sociales, CLACSO.

De Mattos, C. (2016). Financiarización, valorización inmobiliaria del capital y mercantilización de la metamorfosis urbana. Sociologias, (18), 24-52.

Díaz, I. (2013). La gentrificación en la cambiante estructura socioespacial de la ciudad. Revista bibliográfica de geografía y ciencias sociales, XVIII (1030).

Duque, R. (2010). Procesos de gentrificación de cascos antiguos en España: el Albaicín de Granada. Granada: Universidad de Granada.

Ghertner, A. (2015) Why gentrification theory fails in 'much of the world'. City: analysis of urban trends, culture, theory, policy, action, (19), 552-563.

Glass, R. (1964). London, Aspects of Change. Londres: Macgibbon y Kee.

Harvey, D. (1973). Social Justice and the City. España: Siglo XXI.

Havey, D. (1985). The Urbanization of Capital. Baltimore: Johns Hopkins University Press.

Harvey, D. (2013). Ciudades rebeldes. Del derecho de la ciudad a la revolución urbana. Madrid: Akal.

Instituto Nacional de Vivienda y urbanismoINVU. (1983). Plan Regional Metropolitano GAM. Gobierno de la República, San José, Costa Rica.

Janoshcka, M. (2002). El nuevo modelo de ciudad latinoamericana: fragmentación $y$ privatización. Eure, 28 (85), 11-20.

Klotchov, V. (2001). Brevísima Historia De La Planificación Urbana De San José. Ambientico, (99), 4-6.

Láscaris, C. (s.f.). Algunas consideraciones sobre las ciudades en Costa Rica. Recuperado de https://drive.google.com/ drive/folders/0BzzqpghIS_48ZXFsb1NE RzBWckE

Lees, L., Slater, T. y Wyly, E. (2008). Gentrification. London: Routledge.

Lees, L; Shin, H y López-Morales, E. (2015). Global Gentrifications: Uneven Development and Displacement. Bristol: Policy Press.
Lees, L; Shin, H y López-Morales, E. (2016). Planetary Gentrification. Cambridge: Polity Press.

Ley, D. (1996). The new middle classes and the remaking of the central city. Oxford: Oxford University Press.

López, E; Gasic, I y Meza, D. (2014). Captura desigual de renta de suelo y desplazamiento exclusionario. Indicadores generales del proceso de gentrificación en Santiago de Chile, 2000-2012. Cadernos Metrópole, (16), 565-586, São Paulo.

López, E. (2009). Reseña de "Gentrification" de Loretta Lees, Tom Slater y Elvin Wyly. Revista de Geografía Norte Grande, (44), 155-158.

López, E. (2013). Gentrificación en Chile: aportes conceptuales y evidencias para una discusión necesaria. Revista Geografía de Norte Grande, (56), 31-52.

López, E. (2016). Acerca de una gentrificación "planetaria", políticamente útil. Revista INVI, 31 (88), 217-240.

Maloutas, T. (2011). Contextual Diversity in Gentrification Research. Critical Sociology, 38 (1), 33-48.

Matus, C. (2017). Estilos de vida e imaginarios urbanos en nuevos residentes de Lastarria y Bellas Artes: el barrio patrimonial como escenario de diversidad, distinción y movilidad. EURE, (43), 165-186.

Ministerio de Obras Públicas-mopt. (2015). Encuesta de percepción sobre Ciudad Gobierno. Gobierno de la República, San José, Costa Rica.

Ministerio de Vivienda y Asentamientos Humanos (MIVAH) y Cooperación Financiera de la Unión Europea. (2009). Plan Regional Urbano de la Gran Área Metropolitana (PRUGam) 2008-2030. Gobierno de la República, San José, Costa Rica.

Ministerio de Vivienda y Asentamientos Humanos-mivah. (2015). Diagnóstico social de cuarterías. Ministerio de Vivienda y Asentamientos HumanosMIVAH, San José, Costa Rica.

Molina Alfaro, E. (1990). Repercusiones político-organizativas del acuerdo político 
firmado entre los frentes de vivienda y el Estado durante la administración Arias Sánchez. (Tesis para optar por el grado de Máster en Sociología). Universidad de Costa Rica, Ciudad Universitaria Rodrigo Facio, Costa Rica.

Molina, W. (2009). Patrones y flujos de la migración interna en la Gran Área Metropolitana de Costa Rica, en el período 1995-2000. Revista Población y Salud en Mesoamérica, 6 (2).

Molina, W. (2013). Segregación Residencial Socioeconómica en la Gran Área Metropolitana de Costa Rica 19842000. Revista Población y Salud en Mesoamérica, 10 (2).

Mora, M. y Solano, F.(1993). Nuevas tendencias del desarrollo urbano en Costa Rica: el caso del Área Metropolitana de San José. San José: Editorial Alma Máter.

Mora. J. (2003). Análisis del crecimiento urbano de la Gran Área Metropolitana de Costa Rica. Período 1983-2000. (Tesis para optar por el título de grado de Máster en Geografía). Universidad de Costa Rica, San José, Costa Rica.

Municipalidad de San José-MSJ. (2005). Plan Director Urbano de San José (PDUSJ). Municipalidad de San José, San José, Costa Rica.

Municipalidad de San José-MSJ. (2017). Boletín Informativo. Observatorio Municipal, (21), 1-20.

Pérez, M. (1998). La gobernanza urbana y la estrategia centroamericana de desarrollo sostenible. El caso del Área Metropolitana de San José. En Lungo, M. (Comp.) Gobernabilidad urbana en Centroamérica. San José: FLACSO-GURI.

Pérez, M. (2005). Bosque Urbano: proyecto modelo de comunidad verde. El ejemplo de Los Guido-Orowe. Lincoln Institute of Land Policy.

Pérez, M. (2012). Los impactos perversos de la segregación socioespacial en la ciudad de San José. En Pérez M. (Comp.) Avatares del ordenamiento territorial en Costa Rica. San José: Flacso.

Rojo, F. (2016). La gentrificación en los estudios urbanos: una exploración sobre la producción académica de las ciudades. Cadernos Metrópole, 18 (37), 697-719.

Rosero, L. (2002). San José, desierto urbano. Éxodo y envejecimiento de la capital. Recuperado de http://wvw.nacion.com/ ln_ee/2002/mayo/13/opinion.html

Román. J. G. (2013). La incidencia de las políticas públicas de vivienda en la conformación socioespacial del Área Metropolitana de San José. Los casos de Los Cuadros de Goicoechea, Rincón Grande de Pavas y los Guido en Desamparados en el período 1980-2000. (Tesis para optar por el título de grado de Máster en Geografía). Universidad de Costa Rica, San José, Costa Rica.

Rubiales, M. (2014) ¿Medir la gentrificación? Epistemologías, metodologías y herramientas de investigación de carácter cuantitativo y mixto. Contested Cities, Barcelona, España.

Sabatini, F, Robles, M y Vásquez, H. (2009). Gentrificación sin expulsión, o la ciudad latinoamericana en una encrucijada histórica. Revista 180 Arquitectura, Arte y Diseño, 13(24), 18-25.

Salinas, L. (2013). Gentrificación en la ciudad Latinoamericana. El caso de Buenos Aires y Ciudad de México. Geographos. Revista digital para estudiantes de Geografía y Ciencias Sociales, 4 (44), 283-307.

Sassen, S. (1991). The global city, New York, London, Tokio. Princeton: Princeton University Press.

Seligson, M., y Muller, E. (2012). Estabilidad democrática y crisis económica: Costa Rica 1978-1983. Anuario de Estudios Centroamericanos, (16), 71-92.

Sennet, R. (2004). El capitalismo y la ciudad. En Ramos, A. (Coord.), Lo urbano en 
20 autores contemporáneos. Catalunya: Universitat Politècnica de Catalunya, Servicio de Publicaciones.

Smith, N. (2012), La nueva frontera urbana. Ciudad revanchista y gentrificación. Madrid: Traficantes de Sueños, Colección Mapas.

Smith, N. (2015). El mercado contra la ciudad. Sobre globalización, gentrificación y políticas urbanas. Madrid: Traficantes de Sueños, Colección Mapas.
Solow, A. (1949). Proyecto para el desarrollo urbano de la capital de Costa Rica. Washington D.c.: Unión Panamericana.

Swanson, K. (2007). Revanchist urbanism heads south: the regulation of indigenous beggars and street vendors in Ecuador. Antipode, 39, 708-728.

Fecha de ingreso: 06/06/2018 Fecha de aprobación: 09/05/2019 
